\title{
Retinal Nerve Fibre Layer Photography in Glaucomatous Patients
}

\author{
R. A. HITCHINGS, D. POINOOSAWMY, N. POPLAR and G. P. SHETH \\ London
}

\begin{abstract}
Summary
Retinal nerve fibre layer photographs were obtained from sixty-three patients, (113 eyes), attending a glaucoma suspect clinic at Moorfields Eye Hospital. These photographs were studied following masking of the optic disc. Interobserver error was good. Intraobserver error following a six-month 'learning period' showed changes in identification of nerve fibre layer defects occurring as a result of the learning process. Humphrey threshold fields on the 113 eyes were independently scored as abnormals, suspect or normal. A good correlation existed between defects visible on the retinal nerve fibre layer photograph and 'abnormal' visual fields.

Retinal nerve fibre layer photography is possible in glaucoma patients with clear optical media whose pupils permit full mydriasis. It seems to supplement other methods of assessing the early glaucoma patient. It may be of value in sequential follow-up. It would seem to be of little value in the majority of elderly glaucoma patients who would have opacities in the optical media.
\end{abstract}

In 1973 Hoyt and Frisen 'rediscovered' the retinal nerve fibre layer and described how its appearance may change in neuro-ophthalmic disease. ${ }^{\prime}$ They noted that nerve fibre layer defects were to be seen in many glaucoma patients. This observation, although of great interest was largely 'passed by' as being of little value in the management of the average glaucoma patient. The observation was considered too subjective, too difficult to quantify and, in too many cases, obscured by opacities in the optical media to be of routine use.

In 1980 Quigley et al. ${ }^{2}$ were able to demonstrate reproducible semi-quantifiable nerve layer photography with a wide angle camera, producing startling examples of defects in glaucoma patients. They noted nerve fibre loss in a large number of the glaucoma patients studied together with a small number of their normal subjects. Quigley studied the appearance of the nerve fibre layer in primate eyes that had undergone optic nerve damage. ${ }^{3}$ He noted that the visibility of the nerve fibre layer in the normal eye was dependant on, amongst other things, the degree of background pigmentation (the less light reflected from the deeper retina, the better) and the number of remaining nerve fibres (fibre bundles greater than $20 \mu \mathrm{m}$ thick reflect light back to the observer, this means that in the retinal periphery, where the retinal nerve fibre layer is less than $20 \mu \mathrm{m}$ thick the nerve fibre layer will be invisible). Finally, the normal septae existing between fibres correlate well with the striations seen to the nasal and temporal sides of the optic disc. Superiorly, and inferiorly, however, the fibre bundles are thicker, here this relationship is not seen.

From Glaucoma Unit, Moorfields Eye Hospital, London.

Correspondence to: R. A. Hitchings, FRCS, Moorfields Eye Hospital, High Holborn, London WC1V 7AN.

Presented at the Annual Congress of the Ophthalmological Society of the United Kingdom, April 1987. 
Following damage to the optic nerve a number of changes in the retinal nerve fibre layer became visible. ${ }^{3}$ Firstly, however, the damage had to cause $>50$ per cent nerve fibre layer loss. Secondly, subtotal loss of fibres in one segment associated with a lesion involving one section of the nerve will produce well demarcated defects that are easy to see. By contrast diffuse loss will have to wait until 50 per cent of the fibres have gone before becoming visible. Finally, the ' 50 per cent' rule means that even in those areas with a dense retinal nerve fibre defect there is likely to be residual retinal function.

Improvements in photographic technique followed with reports by Sommer on high resolution photography ${ }^{4}$ and the use of cross polarisers 5 and from Airaksinen on the value of the $495 \mathrm{~nm}$ exciter filter to exclude light reflection from the retina deeper to the nerve fibres. ${ }^{6}$ Airaksinen also pointed out the way in which printing techniques helped to improve the final appearance of the layer. In addition Schwartz noted that computer enhancement could provide limited extra help in highlighting these fibre changes. ${ }^{7}$

Using these described techniques a number of clinical studies have been carried out. Sommer et al. reported on the early results of a large study of normal, early and advanced glaucoma subjects, they noted sensitivity and specificity values of 80 per cent and 94 per cent respectively. The greatest source of error came from the presence of 'slit-like' defects and from a general loss of visibilty in the older subject. ${ }^{8}$ Airaksinen was able to correlate the appearance of the retinal nerve fibre layer with optic disc, ${ }^{9}$ diffuse and focal loss of the nerve fibre layer with visual field ${ }^{10-12}$ and colour appreciation. ${ }^{13}$ These studies were, however, carried out in specialised research centres with considerable resources.

Although of interest and important for the insights retinal nerve fibre layer observation gives into the pathophysiology of glaucoma, their relevance to the world of routine glaucoma has to yet to be established. A particular concern has been the loss of visibility of the nerve fibre layer that follows age related lens opacification. This phenomenon would effectively rule out many glaucoma patients, if not immediately, then on follow-up. To that end we have carried out a clinical study investigating the role of the retinal nerve fibre layer in the evaluation and clinical management of glaucoma suspects. We wanted to know whether these photographs would influence assessment of the patient and, in so doing make us alter our management in any way.

\section{Patients and Methods}

Patients attending the glaucoma clinic at Moorfields Eye Hospital with a diagnosis of 'Ocular Hypertension' or who were being followed up because of a suspicious appearance of their optic discs had retinal nerve fibre layer photography according to the method described by Airaksinen and Nieminen. ${ }^{6}$ All patients included in this study fulfilled the following criteria:

(1) I.O.P. $>21 \mathrm{mmHg}$ on more than one occasion OR a suspicious appearance of the optic disc (with a $\mathrm{C} / \mathrm{D}$ ratio $>0.7$ ).

(2) A normal or suspicious Friedmann field. (A suspicious field was defined as a visual field with 2-3 spots having a retinal sensitivity $0.4 \mathrm{log}$ units above threshold).

(3) An open angle and no cause other than primary trabecular damage to account for any elevation of I.O.P.

In addition to the retinal nerve fibre layer photographs all patients had colour photographs of the optic disc to allow for evaluation of the $\mathrm{C} / \mathrm{D}$ ratio and apperance of the neuroretinal rim. A Humphrey Threshold 30-2 test was carried out on at least one occasion.

The retinal nerve fibre photographs were masked by covering the optic disc, so that any changes visible there could not influence the grading of the nerve fibre layer appearance. The fibre appearance was graded as:

(1) 'Normal' with an unbroken series of striations extending around and radiating from the optic disc.

(2) 'Slit-like defects', these were defined as a darker line running parallel to and replacing the visibility of the retinal nerve fibre layer, these were subdivided into (2a) when the defect did not extend to the optic disc, and (2b) where slit-like defects extended to the optic disc (Fig. 1).

(3) 'Wedge shaped defects', where a broad grey zone replaced a number of the retinal nerve fibre layer striations. (A 'groove' kept the same width the length of its visible course, a 'wedge' became wider as it increased in distance from the optic disc.) (3a) was a wedge where some striations were visible within the wedge and (3b) did not have such striations (Fig. 2). 


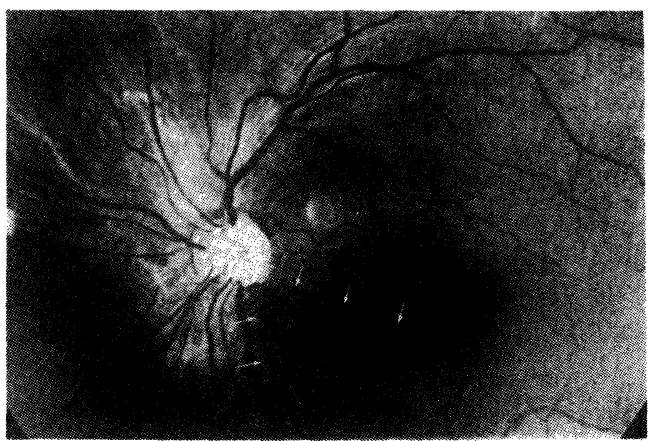

Fig. 1. Nerve fibre layer photographs in a patient with chronic glaucoma having extensive glaucomatous cupping. The inferior retina exhibits a wedge shaped defect (arrows) while there are 'peripheral grooves' (grooves not extending to the disc) in the upper hal fof the retina. The corresponding viual field shows a dense upper altitudinal scotoma

(4) 'Diffuse loss' was considered present if, despite well focused retinal blood vessels the retinal nerve fibre layer was considered 'thin' or indistinct

(5) 'Invisible' when the retinal nerve fibre layer could not be seen in the presence of defocused blood vessels.

The reproducibility of this grading scheme was tested by three observers reading the photographs once and one observer reading the photographs twice with an interval of 6 months between

The photographs were read in a masked fashion with the optic disc blacked out and with no clinical information available.

The results of the nerve fibre analysis were then compared with the results obtained on visual field testing with the Humphrey field analyser. Patients were considered to have an 'abnormal' Humphrey field if one or more spots had a threshold reduced by $10 \mathrm{db}$ or more. The field was considered 'suspicious' if the field had two or more spots reduced by 5-9 db. The results of these comparisons are shown below.

\section{Results}

The patient population consisted of 63 patients, 113 eyes. These patients were regular attenders at the glaucoma clinic at Moorfields Hospital and had undergone repeated visual field tests with the Friedmann analyser. Each patient had undergone a Humphrey test prior to this study.

Interobserver variability was assessed on a sample of 32 eyes. Grades $2 a / 2 b$ and $3 a / 3 b$ were read as 2 and 3 respectively. Agreement was exact between these three observers with the exception of two eyes with out of focus pictures read either as group 4 or 5 .

Interobserver error was assessed by one author (RAH) reading all 113 photographs twice with an interval of 6 months between. The upper and lower halves of the photographs could differ in appearance and have, therefore, been graded separately. The results have been set out in Table I.

The relationship between the retinal nerve fibre appearance and the visual field was studied in two ways; firstly, by comparing the nerve fibre appearance of eyes with 'normal' fields. Secondly, by checking the visual field in those eyes with 'abnormal' retinal nerve fibre layers (grades $2 a$ and $3 b$ above). Patients were graded according to whether they had a defect on the Humphrey 30-2 program against which the retinal nerve fibre layer was compared, or, whether they had a defect on the retinal nerve fibre layer likely to be associated with a defect in the visual field and the corresponding visual field was checked. The results of these two comparisons have been set out in Tables II and III.

\section{Discussion}

This study set out to assess the effectiveness of

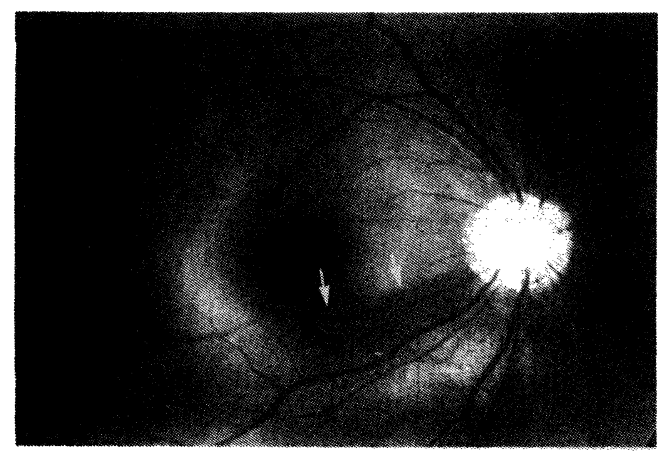

Fig. 2. Retinal nerve fibre layer photograph illustrat ing diffuse loss of fibres in the lower half of the retina. The extent of this area is shown by the arrows. The superior retina has 'grooves' extending to the optic disc. The corresponding visual fields shows a dense upper altitudinal effect and an inferior arcuate scotoma thus matching with the changes showing on the retinal nerve fibre layer photograph.

The illustrations are taken from the eyes of two patients with Chronic Simple Glaucoma. They were used for the purposes of demonstrating changes in the appearance of the retinal nerve fibre layer. 
Table I. Intraobserver error. A comparison of the grades noted in the retinal nerve fibre appearance of 113 eyes (226 hemi-retinas) at the time of two observations six months apart

\begin{tabular}{crrrrrr}
\hline & & \multicolumn{5}{c}{ Rereading grade } \\
\cline { 3 - 7 } Grade & $\begin{array}{c}\text { No. hemi- } \\
\text { retinas }\end{array}$ & 1 & 2 & 3 & 4 & 5 \\
\hline 1 & 70 & 52 & 16 & 1 & 0 & 1 \\
2 & 123 & 73 & 35 & 3 & 0 & 12 \\
3 & 10 & 3 & 3 & 4 & 0 & 0 \\
4 & 0 & 0 & 0 & 0 & 0 & 0 \\
5 & 23 & 4 & 7 & 0 & 0 & 7 \\
\hline Total & 226 & & & & & \\
\hline
\end{tabular}

Table II. A comparison between 'abnormal' fields and the RNFL

11 patients, 13 eyes, had 'abormal fields

2 patients, 2 eyes had a nerve fibre defect corresponding with the visual defect

3 patients, 5 eyes had generalised depression associated with RNFL defects

2 patients, 2 eyes had focal defects associated with 'normal' RNFL

2 patients, 2 eyes had extensive 'upper lid' defects with an intact RNFL

2 patients, 2 eyes had a visual defect with a noncorresponding RNFL defect

Table III. A comparison between 'abnormal' $R N F L$ and the visual field

20 patients, 27 eyes had an 'abnormal' RNFL

5 patients, 7 eyes had 'abnormal' corresponding hemi-fields (and-appeared in Table II above)

1 patient, 2 eyes had tunnel vision in both eyes

14 patients, 18 eyes had 'normal' or 'suspect' fields

retinal nerve analysis in the evaluation of glaucoma suspects. The patient sample consisted of eyes with so-called ocular hypertension, considered to have a normal or suspicious Friedmann field or eyes with large $(>0.7 \mathrm{C} / \mathrm{D})$ optic cups. The patients chosen were considered to have sufficiently clear optical media so that they would provide good pictures of the retinal nerve fibre layer. They came from a patient group where it can be very difficult to establish without doubt the diagnosis of primary open angle glaucoma.

The requirements for good nerve fibre layer photography include, a wide angle camera incoporating an exciter filter set at $495 \mathrm{~nm}$.
The angle width is essential to capture all easily seen nerve fibres, while the filter is essential to highlight the reflectance from the nerve fibres and minimise that from deeper retinal structures. We found a Canon well suited for this purpose. It is essential to see that the optical system of the camera is aligned correctly otherwise unwanted reflexes appear on the film.

The preliminary study of interobserver reproducibility suggested that, with the exception of differentiating between defocused film and diffuse loss it should be possible to correctly categorise the different appearances of the RNFL. The intraobserver study, however, pointed out difficulties in distinguishing between grades $2 \mathrm{a}$ and $2 \mathrm{~b}$.

This is important, for the former is associated with a normal field, the latter with visual defects. However, by adhering to the principle that to be 'significant' the groove needs to reach the margin of the optic disc the grooves so identified will represent focal visual loss.

The patients chosen for this study were considered suitable from the combination of clear optical media and 'early' glaucoma. The former is essential if useful prints are to be obtained. The latter is important because all such patients are debated over as to whether they should be considered for (a lifetime of) antiglaucoma treatment.

The comparison between the visual defects and the retinal nerve fibre defects shown in Table II highlights the difficulties in identifying early visual loss from a single visual field. A close association was found between the two in 7 of the 13 eyes. Only in 4 eyes was the association not clear cut. As in these cases the defects were small, although they met the study criteria for 'abnormality' there was no guarantee that visual loss had occurred. On repeat testing this type of defect may well appear less pronounced.

Seven eyes had significant defects in the RNFL (2a and $3 b$ ) associated with a corresponding visual defect. Eighteen of the remaining 20 eyes had no detected visual loss (the other patient had tunnel vision). Closer spacing of the tested retinal points may identify the site of the retinal sensitivity loss in these cases, even though in some instances it 
would appear necessary to take closely spaced profile perimetric cuts to do so (Heijl, Personal communication).

The identification of early (small) defects in the visual field is of importance in the management of the glaucoma suspect. Because these early defects may be lost in the 'noise' of the method, or lost between the spots tested on the Perimeter, any objective help is to be welcomed. Retinal nerve fibre layer photography is a simple photographic technique, even if it is allied to more complicated film development. In patients with clear optical media it can offer useful ancillary evidence that visual loss may be present, or, perhaps more importantly, evidence that a suspect visual field is not so bad as had been first suspected. The technique can be seen as a supplement to other conventional methods of examination, but it does not supplant them.

\section{References}

${ }^{1}$ Hoyt WF, Frisen L, Newman NM: Fundoscopy of nerve fibre layer defects in glaucoma. Invest Ophthal 1973, 12: 214-29.

${ }^{2}$ Quigley HA, Miller NR, George T: Clinical evaluation of nerve fibre layer atrophy as an indicator of glaucomatous optic nerve damage. Arch Ophthal 1980, 98: $1564-71$.
${ }^{3}$ Quigley HA and Addicks EM: Quantitative studies of retinal nerve fibre layer defects. Arch Ophthal 1982, 100: 807-14.

${ }^{4}$ Sommer A, D’Anna SA, Kues HA, George T: Highresolution photography of the retinal nerve fibre layer. Am J Ophthalmol 1983, 96: 535-9.

${ }^{5}$ Sommer A, Kues AH, D'Anna SA, Arkell S, Robin A: Cross-polarization photography of the nerve fibre layer. Arch Ophthalmol 1984, 102: 864-9.

${ }^{6}$ Airaksinen PJ and Nieminen H: Retinal nerve fibre layer photography in glaucoma. Ophthalmology 1985, 92: 877-9.

${ }^{7}$ Peli E, Hedges TR, Schwartz B: Computerized enhancement of retinal nerve fibre layer. Acta Ophthalmol (Copenh) 1986, 64: 113-22.

${ }^{8}$ Sommer A, Quigley HA, Robin AL, Miller NR, Katz J, Arkell S: Evaluation of nerve fibre layer assessment. Arch Ophthalmol 1984, 102: 176671.

${ }^{9}$ Airaksinen PJ, Drance SM, Schulzer M: Neuroretinal rim area and early glaucoma. $A m J$ Ophthalm ol 1985, 99: 1-4.

${ }^{10}$ Airaksinen PJ and Drance SM: Neuroretinal rim area and retinal nerve fibre layer in glaucoma. Arch Ophthalm ol 1985, 103: 203-4.

${ }^{11}$ Airaksinen PJ, Drance SM, Douglas GR, Schulzer $\mathrm{M}$, Wijsman $\mathrm{K}$ : Visual field and retinal nerve fibre layer comparison in glaucoma. Arch Ophthalmol 1985, 103: 205-7.

${ }^{12}$ Airaksinen PJ, Drance SM, Douglas GR, Mawson DK: Diffuse and localized nerve fibre loss in glaucoma. Am J Ophthalmol 1984, 98: 566-71.

${ }^{13}$ Airaksinen PJ, Lokowski R, Drance SM, Price M: Colour vision and retinal nerve fibre layer in early glaucoma. Am J Ophthalmol 1986, 101: 208-13. 